Besgen (Hrsg.)

Handbuch Führungskräfte 



\title{
Handbuch Führungskräfte
}

Arbeits-, Gesellschafts- und Steuerrecht für Geschäftsführer, Vorstände und leitende Angestellte

\author{
herausgegebenvon
}

\section{Dr. Nicolai Besgen}

bearbeitet von

Dr. Nicolai Besgen

Fachanwalt für Arbeitsrecht, Bonn

Dr. Stephan Dornbusch

Fachanwalt für Steuerrecht, Bonn

Dr. Stefan Drewes

Rechtsanwalt, Bonn

Dr. Theo Kade

Fachanwalt für Arbeitsrecht, Bonn

Mario Knepper

Fachanwalt für Steuerrecht, Bonn

Dr. Christopher Liebscher, LL.M.

Fachanwalt für Arbeitsrecht, Berlin

Dr. Marc Liebscher, LL.M.

Rechtsanwalt, Leipzig

Dr. Andreas Menkel

Fachanwalt für Steuerrecht, Bonn

Dr. Andreas Nadler

Fachanwalt für Arbeitsrecht, Bonn

Dr. Stephan Osnabrügge

Fachanwalt für Arbeitsrecht, Bonn

Dr. Christian Velten

Rechtsanwalt, Frankfurt

Sebastian Witt

Fachanwalt für Arbeitsrecht, Bonn

2012
olls
Verlag
Dr.OttoSchmidt
Köln 
Bibliografische Information der Deutschen Nationalbibliothek

Die Deutsche Nationalbibliothek verzeichnet diese Publikation in der Deutschen Nationalbibliografie; detaillierte bibliografische Daten sind im Internet über http://dnb.d-nb.de abrufbar.

Verlag Dr. Otto Schmidt KG

Gustav-Heinemann-Ufer 58, 50968 Köln

Tel. 02 21/9 37 38-01, Fax 02 21/9 37 38-943

info@otto-schmidt.de

www.otto-schmidt.de

ISBN 978-3-504-42058-1

C2012 by Verlag Dr. Otto Schmidt KG, Köln

Das Werk einschließlich aller seiner Teile ist urheberrechtlich geschützt. Jede Verwertung, die nicht ausdrücklich vom Urheberrechtsgesetz zugelassen ist, bedarf der vorherigen Zustimmung des Verlages. Das gilt insbesondere für Vervielfältigungen, Bearbeitungen, Übersetzungen, Mikroverfilmungen und die Einspeicherung und Verarbeitung in elektronischen Systemen.

Das verwendete Papier ist aus chlorfrei gebleichten Rohstoffen hergestellt, holz- und säurefrei, alterungsbeständig und umweltfreundlich.

Einbandgestaltung: Jan P. Lichtenford, Mettmann

Satz: Schäper, Bonn

Druck und Verarbeitung: Kösel, Krugzell

Printed in Germany 